\title{
Obtaining Clinical Improvement in Amyotrophic Lateral Sclerosis with Stabilization of Progressive Functional Loss: Case Report Presentation and Review
}

\author{
Allen J Orehek ${ }^{*}$ \\ Dementia Prevention Center, USA
}

\begin{abstract}
Background: The patient was given the diagnosis during evaluation at a comprehensive amyotrophic lateral sclerosis (ALS) center by the neurology team. During the year prior to the diagnosis of ALS the patient was evaluated by seven different neurologists over the course of the progressive disease. Coordinated evaluation refined the diagnosis to cryoglobulinemia causing a vasculitis that resulted in severe peripheral neuropathy.

Case presentation: An 86-year-old female in general good health with insidious onset of symptoms that gained her classification qualification for an assigned diagnosis of amyotrophic lateral sclerosis. As frequently associated with a devastating diagnosis the patient and family remained motivated for any additional helpful options.

Conclusion: Presented here is how the patient went from bedbound to assisted ambulation along with the complex medical evaluation, case details, exam findings, and additional medical information that allowed and hindered the patient's ability to recover. ALS remains one of the most devastating diagnosis that a patient could be given and the additional information in this case could be helpful to other patients. Described below is the thought process of a coordinated effort when presented with a diagnosis of exclusion.
\end{abstract}

\section{Keywords}

Amyotrophic lateral sclerosis, Vasculitis, Cryoglobulinemia, Peripheral neuropathy, Hyperviscosity syndrome

\section{Abbreviations}

ALS: Amyotropic Lateral Sclerosis; ESR: Erythrocyte Sedimentation Rate; CBC: Complete Blood Count; ANA: Antinuclear Antibody; TSH: Thyroid Stimulating Hormone; MRI: Magnetic Resonance Imaging; P.O. Per Os: Meaning By Mouth, Orally; EMG: Electro Myography; CT: CAT Scan; CTA: Computed Tomography Angiography; CSF: Cerebro Spinal Fluid; DVT: Deep Vein Thrombosis; 3D: Three Dimensional; LMN: Lower Motor Neuron; UMN: Upper Motor Neuron; IBM: Inclusion Body Myositis; DRG: Diagnosis Related Groups; EHR: Electronic Health Record

\section{Introduction}

The following is a case presentation and review of amyotropic lateral sclerosis (ALS), presented in a detailed timeline where clinical decision making is matched by physical manifestations and accuracy of evaluation outcomes can be identified. As a diagnosis of exclusion with high morbidity and mortality rates ALS outcomes based upon a complete evaluation may provide hope and improvement for those who are assigned such a diagnosis.

\section{Manuscript body}

An elderly female had a progressive loss of neurological function simultaneous with other medical problems. A fluctuation between treatment priority of the neurological symptoms and joint and muscle pain type symptoms occurred based upon patient motivation and severity of the symptoms.
Review of the medical record and history provided that the first presentation was a medical office visit for evaluation of arthralgia and fatigue symptoms more than a year prior to the diagnosis of ALS.

*Corresponding author: Allen J Orehek, Dementia Prevention Center, Montage Mt Road, Moosic, PA 18507 USA

Accepted: December 07, 2020

Published online: December 09, 2020

Citation: Orehek AJ (2020) Obtaining Clinical Improvement in Amyotrophic Lateral Sclerosis with Stabilization of Progressive Functional Loss: Case Report Presentation and Review. J Neurodegener Disord 3(1):77-88 
Citation: Orehek AJ (2020) Obtaining Clinical Improvement in Amyotrophic Lateral Sclerosis with Stabilization of Progressive Functional Loss: Case Report Presentation and Review. J Neurodegener Disord 3(1):77-88

Figure 1: Existing medical history.

-age 65, right breast lumpectomy for stage pT1, ER/PR negative invasive ductal carcinoma treated with radiation.

-age 80 acute myocardial infarction, NSTEMI, resulting in percutaneous coronary intervention and drug eluting stent placed in right coronary artery.

-age 83 colon polyp removed.

-age 84 , coronary artery disease with a drug-eluting stent deployment into left anterior descending coronary artery for a $90 \%$ stenosis.

-age 85, dual chamber permanent pacemaker for symptomatic paroxysmal bradycardia, with first degree AV block and a left anterior fascicular block.

-age 86 2D echo, left atrial diameter $34 \mathrm{~mm}$, global left ventricular ejection fraction 65\%, mild aortic valve sclerosis and mitral insufficiency. Carotid artery vascular study with 1-20\% bilateral stenosis. No evidence of abdominal aortic aneurysm by B-mode imaging of the aorta, velocity was $68 \mathrm{~cm} / \mathrm{sec}$. She had a laparoscopic cholecystectomy and the development of significant neurological disorder.

\section{Background medical and social history}

The patient lived on an operating dairy farm and worked in the insurance industry with five children and 13 grandchildren. No tobacco, alcohol or drug use. After retirement she worked as a volunteer and was assisting a local woman with transportation for chemotherapy at the time of presentation. Stable heart disease and other existing medical history is summarized in Figure 1.

Month 1: The patient's initial complaint was of right knee pain worse than left knee. The initial exam was consistent with crepitus on movement, slight decreased range of motion, pain with movement, no significant muscle weakness, deformity, erythema or swelling found in this ambulatory patient. Orders were for plain view x-rays, over the counter medications and referral to physical therapy. With the current treatment plan the patient reported the knee symptoms were no worse but she did start to notice some weakness and myalgias of the legs.

Month 2: Interpretation of the X-rays of the knees showed minimal changes to joint space with only a slight narrowing, normal anatomical alignment with minimal bone spur and no fractures. Examination showed persistent pain with movement of the bilateral knee joints. Meloxicam $7.5 \mathrm{mg}$ P.O. daily was added but provided no relief after a two week trial. Following no response to medications, the left and right knees were injected by sterile technique with $80 \mathrm{mg}$ of triamcinolone acetonide and orders for continued use of physical therapy for the muscle weakness. Two weeks later the patient reported muscle weakness in both legs with increase of myalgia symptoms associated with pain in right knee radiating up into the quadriceps muscles. Symptoms in the left leg were generalized to the left knee. There was no significant response to the treatment plan of medications, the injections and physical therapy. Orders included blood work for further investigation. The patients rheumatoid factor was elevated at $45.0 \mathrm{IU} / \mathrm{mL}$, and the remaining labs within normal reference range: anti-CCP 8 units (cyclic citrullinated peptide), Aldolase $7.3 \mathrm{U} / \mathrm{L}$, ESR $18 \mathrm{~mm} / \mathrm{hr}$. creatinine $1.10 \mathrm{mg} / \mathrm{dL}, \mathrm{CBC}$, ANA (antinuculear antibody), uric acid, TSH, C-reactive protein 0.34 $\mathrm{mg} / \mathrm{L}$, Lyme IgG/IgM negative and serum chemistry normal. Joint aspiration was considered but postponed due to limited fluid across joint on exam.

Month 3: The patient remained completely independent in her house hold activities and driving. The medical evaluation revealed development of new bilateral shoulder area pain and myalgias with a further progression of the right leg pain from hip to knee. Her arthralgia symptoms persisted in the bilateral knees. The patient's pain took on an element of being pervasive from the hips down, yet remained localized to the area of the knee mostly. Muscle weakness and progressive fatigue continued to be noted without changes in reflex or sensation. The differential of the muscle weakness included skeletal muscle loss from lack of use secondary to arthralgia and myalgia of the limbs. The combination of treatment response failure along with the complex presentation prompted a rheumatology consultation for additional evaluation.

Month 4: Pending the availability of the rheumatology consultation the meloxicam was discontinued due to creatinine increase from $1.1 \mathrm{mg} / \mathrm{dL}$ to $1.4 \mathrm{mg} / \mathrm{dL}$. The medication was changed to prednisone $30 \mathrm{mg}$ daily for 5 days then decreasing to a dose of $20 \mathrm{mg}$ daily until specialist evaluation. Due to the abnormal neurological exam and weakness an MRI of the brain was ordered to rule out a central nervous system related etiology. As the patient had a pacemaker placed for sick sinus syndrome and pauses in the past, while compatible with MRI, the leads were reported as not compatible with an MRI scan. The orders were changed to a CAT scan of head.

Month 5: Rheumatology consultation provided a diagnosis of rheumatoid arthritis failing conservative measures of NSAIDS, meloxicam, therapy and joint injections. The Rheumatologist reported that the patient had some relief in symptoms on the prednisone $20 \mathrm{mg}$ daily over the last four weeks but was still having exacerbations so severe they prevented her from normal house hold activities. As the patient was experiencing a progressive decline across a number of different measurable areas treatment with a disease-modifying anti-rheumatic drug was initiated. The patient was given hydroxychloroquine (Plaquenil) 400 m.g. P.O. Q.D. as an attempt to decrease pain and tenderness. Prednisone was tapered to off over a 30 day period of time. This patient had an eye exam during the previous year and was free from any retinopathy.

Month 6: Early into the new treatment with hydroxychloroquine the patient developed dyspnea and acute chest pain. She presented to local emergency department for evaluation. EKG showed a paced rhythm, chest x-ray without acute 
Citation: Orehek AJ (2020) Obtaining Clinical Improvement in Amyotrophic Lateral Sclerosis with Stabilization of Progressive Functional Loss: Case Report Presentation and Review. J Neurodegener Disord 3(1):77-88

Figure 2: Radiology data.

X-ray of cervical spine: marked thoracic kyphosis unable to evaluate right cervical neuroforamina. X-ray of thoracic spine: several mild wedge compression fracture deformities unchanged from year prior, normal bone mineralization. X-ray lumbar spine: views limited by over penetration and positioning, moderate levo lumbar scoliosis, disc space narrowing at L1-L2, Moderate to severe disc space narrowing at L2-L3, moderate disc space narrowing at L3-L4, and severe disc space narrowing at L4-L5. Serum chemistry normal. Complete blood count normal. ANA negative, ESR 38 mm/hr, TSH normal, cpk 129 u/l, HS-CRP 0.48 mg/l, C3 138, C4 38.4, Hgba1c 5.1, and calcium 9.2 mg/dl.

Figure 3: CT imaging.

Computerized tomography (CT) of cervical spine: evaluation limited due to positioning due to curvature, evaluated neural foramina were patent. CT of thoracic spine: posterior osteophytic ridging and disc bulging at T8-T9 and T9-T10. CT of lumbar spine: L1-L2 mild disc bulging, L2-L3 facet arthrosis bilaterally with minimal bilateral neural foraminal narrowing. L4-L5 moderate bilateral neural foraminal narrowing. Moderate with left greater than right neural foraminal narrowing at L5-S1 and unroofing of the intervertebral disc.

abnormality, creatinine $0.75 \mathrm{mg} / \mathrm{dl}$, troponin $\mathrm{T} 0.60 \mathrm{ng} / \mathrm{mL}$ (normal reference 0.01-0.04 ng/mL), CK-MB $6.3 \mathrm{ng} / \mathrm{ml}$ (range $0.0-3.6 \mathrm{ng} / \mathrm{ml})$. The patient was released from the emergency department with the assigned diagnosis of costochondritis and chest pain syndrome from medication. The emergency department indicated the side effect of the hydroxychloroquine causing a chest pain syndrome and she was discharged. Medical treatment changed to methotrexate $7.5 \mathrm{mg}$ once a week with folic acid $1 \mathrm{mg}$ Q.D.

Month 7: She presented complaining of a left leg spider bite, yet she did not recall any contact with arachnids. Exam of the skin lesion showed a $15 \mathrm{~mm}$ erythematous papule with central purpuric area. A confusing presentation as there was no indication of any "bite" mark within the skin lesion. This atypical presentation for arachnid bite had surrounding erythema suggestive of early cellulitus and cephalexin $500 \mathrm{~m} . \mathrm{g}$. Q.I.D. was provided as the patient was on potential immune altering medication of methotrexate. As the confidence in this being an arachnid or insect bite was with great presumption of the situation the medical evaluation was expanded to evaluate for a vascular related lesion. Consultation with vascular specialist was arranged for arterial duplex scan of the leg to rule out arterial etiology for skin lesion and the plan was for biopsy if the lesion showed progression. A week later the lesion transformed to develop a central area of ulcer and a full thickness defect of the skin. The treatment was adjusted to wound cleaning twice a day with further observation. As the lesion had progressed toward an eventual healing by secondary intention without signs of untreated infection conservative measures were suggested in this complex patient who had had additional medical priorities. The patient reported a continued progression of myalgia, arthralgia, and muscle weakness. Physical exam revealed she was developing atrophy of the thenar eminence of the left hand associated with a wrist extension weakness. Plan included need of electromyography (EMG) and neurology consultation. The rheumatology specialist was called case reviewed for the worsening of her status. The methotrexate was changed from oral to subcutaneous injection. A general loss of function left her unable to drive and now needed to use a wheeled walker for ambulation. The patient canceled the arterial studies because of changes in having to rely on transportation, healing of the leg wound, continued weakness and other medical priorities related to the joint and neurological symptoms.
Month 8: After six weekly injections of subcutaneous methotrexate $10 \mathrm{mg}$. the patient's exam showed a progressive loss of muscle mass and strength in both lower extremities and left arm. Associated myalgia was noted in all limbs. With a degree of daily variability the patient reported some good days and some days that were much worse with the main consistent problem being the pain in the knees and wrists. The result of an unclear response to the current medical treatment plan included expanded evaluation of radiculopathy with imaging, expanded lab work, second rheumatology consultation and electromyography (EMG) of arms and legs. Figure 2 contains the details and results.

Month 9: The patient had continued progression of symptoms along with additional muscle atrophy. She reported a sensation of water dripping down the right leg. Medical evaluation by an additional rheumatology team found weakness of the legs and hands causing an inability for ambulation and difficulty standing. The left hand developed flexion deformities of the index and long finger. Severe muscle wasting of intrinsic muscles of the left hand was also identified. Deep tendon reflexes were reported as normal. The evaluation suggested a cervical myelopathy type process associated with lumbar stenosis (Figure 3). The recommendations to find the etiology for balance and weakness associated with muscle atrophy were for CT myelogram, labs, and neurosurgical consultation.

Month 10: With the findings of limited view in the cervical spine and moderate bilateral foraminal narrowing in the lumbar spine a neurosurgical consultation was placed with the team unable to offer any surgical resolution. Electromyography (EMG) testing results revealed cervical and lumbosacral polyradiculopathies affecting nearly every level sampled (Figure 4).

Month 11: Additional neurology consultation suggested CT myelogram to evaluate cervical and lumbar spine. The CT myelogram was completed with finding of severe disc space narrowing at L4-L5 with osteophyte encroachment of bilateral neural foramina resulting in request for neuroendoscopic evaluation with additional neurosurgical team Figure 5, evaluating the potential of concurrent cervical and lumbar radiculopathies. CT of brain reviewed by two radiology teams was reported as generalized cerebral edema with effacement of the cortical sulci and focal loss of gray-white differentiation in the left parietal apex. With this finding suggesting a severe 
Figure 4: Electromyography results.

Electromyography (EMG): Right median and ulnar motor latencies were mildly prolonged. The median motor conduction velocity and amplitudes were normal. The ulnar amplitude was reduced at all stimulation points and the motor conduction velocity was slowed across the elbow. The median and ulnar palmar sensory latencies were mildly prolonged with normal amplitudes. The radial sensory study was normal. The right tibial and peroneal motor latencies were normal with low amplitudes. The peroneal motor conduction velocity was normal while the tibial was moderately slowed. A widespread spontaneous activity with fibrillation and PSWs seen in muscles of all four extremities. Fasciculations were only definitely seen in the left triceps muscle. Chronic neuropathic motor units with reduced recruitment were seen in nearly every muscle to a variable degree with the finding more prominent in the left leg and left arm than the right. Study noted that the patient's limbs felt cold.

Figure 5: CT myelogram.

CT myelogram: Cervical myelogram was interpreted as no disc herniation, canal stenosis or foraminal compromise. The throacic spine: 12 degree dextroconvex scoliosis centered at T8-T9 with associated small disc bulges, but no canal or foraminal stenosis. Lumbar spine: 18 degrees of levoconvex scoliosis centered on L3. fracture of L1 involving the superior endplate and verterbral body. An associated buckle of the posterior vertebral body margin and retropulsion of the bony elements by $5 \mathrm{~mm}$. Severe disc space narrowing at L4-L5 with osteophyte encroachment of bilateral neural foramina.

Figure 6: Hospital employed physician neurological exam.

Hospital neurologist exam: Patient describing fasciculations in the left arm. The patient had left side weakness, weakness of the right hip flexor, decreased bulk on left side, mildly increased tone in the upper extremity with no muscle fasciculations. Deep tendon reflexes $2+$ in the upper extremities and hypoactive in the lower, finger to nose testing intact bilaterally, sensory grossly intact to light touch and pinprick with some decreased vibration in great toes bilaterally. The proprioception in great toes was intact. There were no signs or symptoms of encephalopathy.

Figure 7: CTA of neck and brain.

CTA from aortic arch through brain was completed: the bilateral carotid arteries and vertebral arteries patent at origins and in their extracranial course. The left and right internal carotids were grossly patent with mild atherosclerotic changes noted within the intracranial arteries. There was no intraluminal cut-off or significant arterial occlusive disease. Adequate opacification of the M1 segments of the bilateral middle cerebral arteries along with perfusion of the distal branches. There were no outpouchings to suggest aneurysm formation. Brain tissue showed a redemonstration of diffuse effacement of the cortical sulci and generalized cerebral edema.

Figure 8: Electromyography results.

Electromyography: Fasciculations were noted at $1+$ in bilateral thoracic paraspinals, left triceps brachii, left flexor carpi ulnaris, left first dorsal interosseous. EMG for 22 muscles: normal in $\mathrm{R}$ deltoid, $\mathrm{R}$ triceps brachii, right pronator teres, left deltoid, $r$ vastus medialis, $r$ vastus lateralis, $r$ tibialis anterior, left vastus lateralis, left tibialis anterior, I tibialis posterior. the study was abnormal in 12 muscles with abnormal spontaneous activity found in left thoracic paraspinals, right thoracic paraspinals, right biceps brachii, right first dorsal interosseous, left triceps brachii, left brachialis, left flexor carpi ulnaris, left first dorsal interosseous, right tibialis posterior, right gastrocnemius medical head, left vastus medialis, left gastrocnemius medial head. The "MUP' waveform abnormality was found in R first dorsal interosseous, left triceps brachii, left flexor carpi ulnaris, left first dorsal interosseous, right tibialis posterior, right gastrocnemius medical head, left vastus medialis, left gastrocnemius medial head. Abnormal interferrence pattern was found in right first dorsal interosseous, left triceps brachii, left flexor carpi ulnaris, left first dorsal interosseous, right tibialis posterior, right gastrocnemius medial head, left vastus medialis and left gastrocnemius medial head. Abnormal study limited by limb temperature and evidence of diffuse muscle denervation and fasciculations.

encephalopathy the patient was admitted to local hospital.

During this hospitalization the patient was evaluated by new neurology team Figure 6. The neurology consultation for the abnormal outpatient CT of brain with concern for cerebral edema found that there were no explanations for the CT of brain findings. An additional CTA of the brain was completed Figure 7. The assessment of the brain CT findings and the progressive weakness in the left greater than right upper extremities and bilateral lower extremities remained unclear. The hospital based plan was for observation and consideration to a repeat EMG as outpatient for a diagnosis of probable amyotrophic lateral sclerosis.

Month 12: The third neurology consultation was obtained.
The observations included left hand and arm weakness with atrophy associated with brisk arm and leg reflexes. Electromyography from two months prior was reviewed and found "cervical and lumbosacral polyradiculopathy affecting nearly every level sampled, limited by suboptimal limb temperature". The patient provided symptoms of feeling achy, stiff, weak, and sluggish with episodic arm and leg cramps with description of muscle fasciculations throughout. Electromyography was repeated Figure 8 and labs were obtained. Results of the blood work showed a normal range of GM1antibody, calcium, CK, and parathyroid hormone. The patient was diagnosed with definite ALS and referred to a comprehensive ALS center for management.

Evaluation by the neuroendoscopic neurosurgical team 
Citation: Orehek AJ (2020) Obtaining Clinical Improvement in Amyotrophic Lateral Sclerosis with Stabilization of Progressive Functional Loss: Case Report Presentation and Review. J Neurodegener Disord 3(1):77-88

Figure 9: Expanded medical investigation.

Patient had no geographic access to nerve biopsy as her medical record included the diagnosis of amyotropic lateral sclerosis [1]. Office based skin biopsy indications were reviewed and considered [2]. There was no geographic lab that would offer immunostaining with antiprotein-gene-product 9.5 (PGP 9.5) antibodies and then examine with immunohistochemical methods. Urine free Kappa Lt Chains 5.2 $\mathrm{mg} / \mathrm{L}$ (range 1.35-24.19), Urine Free Lambda Lt Chains $0.20 \mathrm{mg} / \mathrm{L}$ (range 0.24 - 6.66), Kappa/Lambda 26.00H (range 2.04 - 10.37), hepatitis A IgM: nonreactive, hepatitis $B$ core: nonreactive, hepatitis $B$ surface $A B$ : nonreactive, hepatitis $B$ surface $A G$ : nonreactive, hepatitis $C A B$ : nonreactive, SPEP/SIEP: normal with no M-spike observed, CCP IGG/IGA AB: 9 units (range: 0-19 units), Lyme disease Western blot IgG: Band kD/intensity \% of cut-off: 93/091, 66/027, 58/030, 41/111, 34/052, Lyme disease Western blot IgM: Band kD/intensity \% cut-off: 41/052, 34/060, bartonella henselae IgG positive index 2.05 (range 0.01 - 0.02), negative IgG/IgM titers for anapasma phagocytophilum, babesia microti, ehrlichia chaffeensis, rickettsia rickettsii, borrelia afzelii, borelia garinii, borelia lonestari, babesia WA1, ANA negative, ESR $34 \mathrm{~mm} / \mathrm{hr}$, TSH normal, cpk 119 u/l, HS-CRP $1.34 \mathrm{mg} / \mathrm{l}$, magnesium $2.5 \mathrm{mg} / \mathrm{dl}, \mathrm{C} 3$ 133, C4 28.4, rhumatoid factor 27.2 (range 0-15 IU/ml), Hgba1c 5.1, calcium $9.6 \mathrm{mg} / \mathrm{dl}$, PTH 64.9, vitamin C $1.2 \mathrm{mg} / \mathrm{dl}$ (range $0.2-2.0 \mathrm{mg} / \mathrm{dl}$ ), normal anti-cardiolipin antibody IgG, IgM, and IgA, normal antiphospholipid IgG, IgM, and IgA, Factor V leiden R506Q negative, single MTHFR C677T mutation, Factor II G20210A negative, homocystine $13.4 \mathrm{umol} / \mathrm{l}$ (range 3.2-10.7 umol/I), C-ANCA and P-ANCA normal, and aldolase $5.8 \mathrm{u} / \mathrm{l}$ (range 3.3 - $10.3 \mathrm{u} / \mathrm{l}$ ).

Figure 10: Comprehensive ALS center data review for diagnosis of ALS.

Seated vital capacity of $2.08 \mathrm{~L}, 90$ percent predicted, review of history, additional information of 4 falls months before that could have caused the L1 burst fracture, review of CT myelogram, review of EMG data from two separate neurologists over time, Lyme test results, and the plan was treatment of upper motor neuron disorder of cervical and lumbosacral along with lower motor neuron disorder of cervical, thoracic and lumbosacral. Medication of riluzole was given along with follow-up for amyotrophic lateral sclerosis management.

Figure 11: Comprehensive ALS center lab investigation and functional status.

Labs: CRP non-cardiac less than $.10 \mathrm{mg} / \mathrm{dl}$, ESR 23, CK 114, creatinine $0.69 \mathrm{mg} / \mathrm{dl}$, calcium 10.4, hospital bed, power wheelchair, bedside commode, Hoyer lift and social work follow-up. Evaluation of functional rating scale: score 36 (functional rating scale: speech 4, salivation 4 , swallowing 4 , handwriting 3, oral feeding 3 , dressing and hygiene 2 , turning in bed 2, walking 2 , climbing stairs 0 , breathing 444 . total score 36)

for the myelogram findings of severe disc space narrowing at L4-L5 with osteophyte encroachment of bilateral neural foramina was completed with no surgical options available. The patient had a new complaint of hair loss with hair falling out in clumps. With endoscopic neurosurgical team having no explanation for the neuropathy and the neurology teams assigning a diagnosis of ALS additional medical investigation was expanded along with a request for nerve or skin biopsy (Figure 9) [1,2].

Month 13: Evaluation noted an elevated urine kappa/ lambda and equivocal range testing for Lyme disease western blot as shown in Figure 9. A neurology consultation at a comprehensive ALS center was obtained. The evaluation at the tertiary neurology center found diagnosis of ALS (Figure 10).

Month 14: The patient was seen by the tertiary comprehensive ALS center for follow-up by and found to have progressive decline. Additional orders and results are included in Figure 11. The Patient and family requested of the comprehensive ALS center a complete review of history, testing results, and current examination for definiteness of diagnosis. A review of the prior medical documentation, labs and EMG studies, in the setting of progressive upper and lower motor neuron disorder, was a diagnosis of definite amyotrophic lateral sclerosis. Review of the records indicate the patient's criteria for diagnosis of definite ALS were: 1) Clinical presentation of a progressive loss of neuron function; 2) The EMG findings of fasciculations; 3 ) The clinical decision of the team; and 4) Referring neurology teams identification of amyotrophic lateral sclerosis.

Month 15: Patient returned to primary medical team for extensive case review. The medical evaluation focused on investigation of a large fiber/small fiber neuropathy in this patient who was currently assigned a diagnosis of ALS by six different neurology teams and a comprehensive ALS center. Open discussion with the family related to the topic of "not needing to order every test but it is necessary to order the correct test" took place. The evaluation was expanded to include: Ganglioside antibody, CSF PCR to evaluate for infectious (example: Lyme disease) or other inflammatory (example: carcinomatous root involvement), cryoglobulinema evaluation, bone survey due to abnormal kappa/lambda, and amyloid testing. Sural nerve biopsy was agreed upon but there was no local geographic access to this procedure and its priority shifted to lower on the list [3]. Prior documentation details were reviewed for ruling out many etiology (HIV, Borreliosis, alcohol, drug and toxin, connective tissue, diabetes, chronic renal failure, vitamin deficiency, and endocrine) [4]. The findings are reviewed in Figure 12.

Month 16: The patient presented to the office urgently with left leg swelling. A stat venous ultrasound of the leg showed massive deep vein thrombosis (DVT). Clot was identified as non compressible, absent color flow, echo filled Doppler signals in the left external iliac, common femoral, femoral and popliteal veins. This was consistent with extensive occlusive DVT of left lower extremity. The patient was sent directly to the emergency room from testing facility. She was hospitalized and provided care by the hospital based medical care team. The hospital electronic medical record contained extensive data and testing including the results of cryoglobulin positive serum at the time of admission. (type II mixed cryoglobulins present consisting of monoclonal IGG as well 
Citation: Orehek AJ (2020) Obtaining Clinical Improvement in Amyotrophic Lateral Sclerosis with Stabilization of Progressive Functional Loss: Case Report Presentation and Review. J Neurodegener Disord 3(1):77-88

Figure 12: Primary medical team expanded investigation.

Cryoglobulin positive (+) with type II mixed cryoglobulins present consisting of monoclonal IGG as well as polyclonal immunoglobulins. Amyloidosis - improper specimen obtained at hospital lab, Cerebrospinal fluid gram stain: rare polymorphonuclear leukocytes and no organisms. CSF cell count with elevations noted as: RBC 51 cells $/ \mathrm{mm}^{3}$, fluid wbc 1 cells $/ \mathrm{mm}^{3}$, neutrophils 0.1 cells $/ \mathrm{mm}^{3}$, lymphs 0.9 cells $/ \mathrm{mm}^{3}$, monocytes 0.1 cells $/ \mathrm{mm}^{3}$. CSF glucose $67 \mathrm{mg} / \mathrm{dL}$, protein $36 \mathrm{mg} / \mathrm{dL}$, CSF A-beta $42328.75 \mathrm{pg} / \mathrm{mL}, \mathrm{T}-\mathrm{Tau} 411.5 \mathrm{pg} / \mathrm{mL}, \mathrm{P}$-Tau $77.9 \mathrm{pg} / \mathrm{mL}$, A-beta 42 to T-tau index (ATI) 0.45 with interpretation: Alzheimer Disease. Ganglioside GM-1 antibody (IgG) $<1: 800$ titer (normal),Vitamin E $12.2 \mathrm{ug} / \mathrm{mL}$, urine arsenic $10 \mathrm{mcg} / \mathrm{g} \mathrm{cr}$ (normal less than $35 \mathrm{mcg} / \mathrm{g}$ creatinine), urine mercury $<4 \mathrm{mcg} / \mathrm{L}$, urine lead $<10$ $\mathrm{mcg} / \mathrm{L}$, random urine creatinine $112.8 \mathrm{mg} / \mathrm{dL}$, Elevated uroporphyrins I at $25.0 \mathrm{mcg} / \mathrm{g}$ creatinine (3.1 to $18.2 \mathrm{mcg} / \mathrm{g}$ creat), uroporphyrins III $3.4 \mathrm{mcg} / \mathrm{g}$ creatinine (normal), heptacarboxyporphyrin $1.0 \mathrm{mcg} / \mathrm{g}$ creatinine (normal), hexacarboxyporphyrin, pentacarboxyporphyrin, coproporphyrin, coproporphyrin III (normal).

Figure 13: Cognitive testing.

\begin{abstract}
Evaluation for any cognitive dysfunction, including testing of executive functioning while avoiding any potential copyright violations using The Montreal Cognitive Assessment. [5-7]

Visuospatial and executive functioning (alternating trail making): 1 point, Visuoconstructional skills (cube): Without adjustment for the neurological challenges of motor function, 0 point, Visuoconstructional skill (clock): 3 points, Naming: 3 points, Memory (end of test word recall without cues): 5 points, Attention forward digit span: 1 point, Attention backward digit span (0 Point), Vigilance: 1 point, Attention serial 7s: 3 points, Language sentence repetition: 2 points, Language fluency: (Patient named 15 words) 1 point, Abstraction: 2 points, Delayed recall (Short-term memory): 5 points without use of cues, Orientation: 6 points. TOTAL: 28/30 points.
\end{abstract}

Addenbrooke's Cognitive Examination, using the ACE-III version to avoid any potential copyright violations was utilized. This test focuses on five cognitive domains.[8] Attention (orientation): 10 points, Attention (registration): 3 points, Attention (serial 7): 5 points, Memory (recall): 3 points, Verbal Fluency (letters): 5/7 points, Verbal Fluency (animals) 5/7 points, Memory (anterograde memory): 7 points, Memory (retrograde memory) 2/4 points, Language (comprehension): 3 points, Language (sentence writing): 2 points, Language (single word repetition): 2 points, Language (proverb repetition): 2 points, Language (object naming): 11/12 points with misidentification of kangaroo, Language (comprehension): $3 / 4$ points with marsupial being missed, Language (reading): 1 point, Visuospatial ability (intersecting infinity loops): 1 point, Visuospatial ability (3D wire cube): 1/2 points with neurological motor function being a noted factor, Visuospatial ability (clock): $4 / 5$ points with aspects of motor dysfunction being noted and length of clock hands being transposed, Perceptual ability (counting dots): 4 points, Perceptual ability (identifying letters): 4 points, Memory (address recall): 6/7 points +5 points. TOTAL ACE-III score: Attention 18/18, Memory 23/26, Fluency 10/14, Language 24/26, Visuospatial 14/16, TOTAL ACE-III score: 89/100.

Note: While testing using the Addenbrooke's Cognitive Examination it was clear that the test version (English Version A 2012) could have been replaced with USA Version ACE-R (2005), to avoid some content issues. While the patient did recall the address of 73 Orchard Close, the questions related to Prime Minister were unrelated, and not growing up in Australia failure to identify a marsupial could be explained.

Mini-Mental Status Examination testing was used but the score (29/30) was not recorded because of unclear and uncertain copyright issues related to testing use and publication of testing results.[9] Testing for mental impairment in the elderly with Abbreviated Mental Test.[10] Completion of the questionnaire resulted in a score 10/10. Finally, the General Practitioner Assessment of Cognition Test, [11] a free to use test for clinicians and researchers resulted in a score $9 / 9$.

as polyclonal immunoglobulins). The patient was evaluated by the hospital neurology care providers and testing ordered was a CT of the brain with a diagnosis of ALS. Hospital vascular surgery evaluated the patient and recommended: leg elevation with anticoagulation. Hospital hematology evaluation listed DVT secondary to ALS and suggested anticoagulation with heparin and warfarin. During hospitalization the patient lost ability for ambulation and was discharged to home with enoxaparin sodium $80 \mathrm{mg}$ subcutaneous twice a day, warfarin $5 \mathrm{mg}$ daily and was continued on riluzole.

Month 17: House call was made to the patient for review of the hospital stay for the new DVT, the findings of cryogloblemia and discuss additional treatment options. A generalized progressive decline was found with the patient complaints of fasciculations, myalgia, arthralgia, and muscle weakness. Her exam showed swelling in the left leg with cool temperature, diffuse progressive muscle motor function and worse atrophy of left arm. Overall condition and strength were significantly worse since last medical evaluation. Patient and family remained motivated and medical treatment plan was arranged. Treatment options for a diagnosis of type II mixed cryoglobulinemia (monoclonal IgG) resulting in neuropathy from cryoglobulinemia vasculitis causing the described complex patient presentation was initiated. With the findings in the CSF indicating A-beta 42 to T-tau index (ATI) of 0.45 , additional cognitive testing was completed shown in Figure 13 [5-11] (Illustration 1).

Month 18: The patient surmounted treatment initiation for cryoglobulinia vasculitis as discussed below and was admitted to the hospital under the care of an independent rheumatologist. For three days the patient received methylprednisolone $1000 \mathrm{mg}$ intravenously. The riluzole was discontinued. She reported improvement in joint symptoms after the second day and expressed that she did not realized they were as symptomatic prior to treatment. After three days of intravenous steroids the patient was given Rituximab 1000 mg intravenously, which was repeated two weeks after discharge as an outpatient. Oral prednisone $30 \mathrm{mg}$ PO BID and Dapsone $25 \mathrm{mg}$ BID for Pneumocystis jiroveci prophylaxis were added to her medical management.

Month 19: Two weeks after hospital treatment of cryo- 

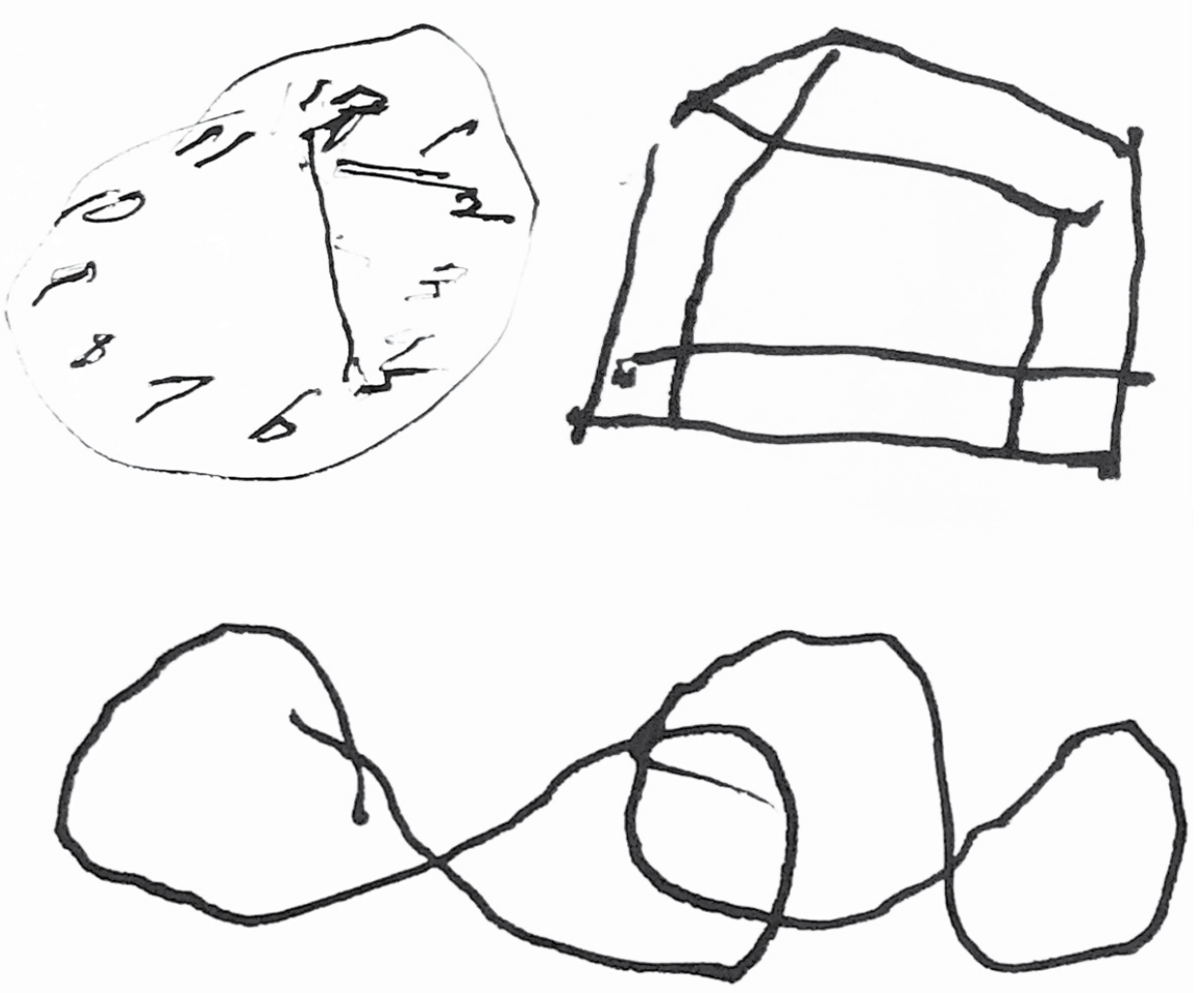

Illustration 1: Hand drawing by patient diagnosed with amyotrophic lateral sclerosis during neuropsychological testing.

Note: During testing the patient was experiencing significant motor dysfunction related to cryoglobulinemia causing vasculitis resulting in vasculitic neuropathy. (Visuospatial ability: Intersecting infinity loops, 3D wire cube, clock).

globulinemia vasculitis the patient was able to take three assisted steps. Over the next month she was found to have improvement of motor function of left wrist flexors and a discontinuation of fasciculation. Limited access to physical therapy and occupational therapy in her house reduced muscle motor recovery progress. The patient was seen and evaluated by independent hematologist who agreed with treatment for Type II mixed cryoglobulinemia with monoclonal IgG including Rituxan/rituximab, steroid taper therapy, and warfarin anticoagulation for the hypercoagulable aspect of the case.

Month 20: After several weeks of awaiting insurance approval, the patient was admitted from home to a physical therapy rehabilitation facility. During inpatient rehabilitation the patient was able to progress to 80 feet of ambulation with a ZeroG gait and balance system and forty feet twice daily with walker assistance [12].

Month 21: Patient reported no additional fasciculations and no additional loss of motor function. An infusion of rituximab 1000 mg intravenously every six months was continued. Due to the amount of motor nerve damage associated with atrophy there was a plateau of any additional improvement in physical activity.

Month 24-28: Patient reported a lifestyle with adjustments to the physical limitations on activity of daily living. There were no additional motor or sensory loss and no new symptoms since the time of discharge from the inpatient physical rehabilitation center. She was utilizing molded ankle-foot orthosis on left leg and assisted walking with roller walker on single level of her home. The patient's impression was if she had more inpatient therapy available, she would be making better progress.

Month 29: The Patient's activities of daily living became more difficult to manage at home, and without any access to insurance covered services for rehabilitation due to "non qualified criteria", she was admitted to rehabilitation facility paying \$252/day for her services.

Months 30-35: The Patient had no additional functional loss or gains. She had a number of falls due to weakness and lack of coordination. Anticoagulation with warfarin for hyperviscosity syndrome and massive DVT was continued along with routine medications. She had no access to additional cryoglobulinemia vasculitis treatment other than the rituximab infusions.

Month 36: The Patient expired during an outbreak of SARS-CoV-2 in the facility.

\section{Discussion}

Defining, describing and diagnosing amyotrophic lateral sclerosis

A diagnosis of amyotrophic lateral sclerosis (ALS) can be described as associated with a number of different defini- 
Citation: Orehek AJ (2020) Obtaining Clinical Improvement in Amyotrophic Lateral Sclerosis with Stabilization of Progressive Functional Loss: Case Report Presentation and Review. J Neurodegener Disord 3(1):77-88

tions. Most definitions will include the diagnosis being based upon clinical presentation of upper motor neuron and lower motor neuron signs associated with progressive aspects in the absence of alternate explanations. As there is no single test that can confirm or exclude the diagnosis of ALS it remains a diagnosis of exclusion. Definition 1: ALS has been described as a progressive, degenerative motor neuron disease of unknown cause. Muscle atrophy and spasticity in limb and bulbar muscles result in weakness and loss of ambulation, oropharyngeal dysfunction, weight loss, and ultimately respiratory failure [13]. Definition 2: ALS is an idiopathic, fatal neurodegenerative disease of the human motor system [14]. Definition 3: ALS is a progressive, paralytic disorder characterized by degeneration of motor neurons in the brain and spinal cord. It begins insidiously with focal weakness but spreads relentlessly to involve most muscles, including the diaphragm. Typically, death due to respiratory paralysis occurs in 3 to 5 years [15].

Diagnostic criteria have been developed to arrange a set of findings that will fit certain criteria to assign a diagnosis of ALS. Contemporarily the revised El Escorial Criteria and the Awaji Criteria remain widely used diagnostic criteria for ALS [16]. The El Escorial criteria use signs of upper motor neuron and lower motor neuron dysfunction to assign levels of certainty of the diagnosis. El Escorial revised criteria assigning a classification of Clinically Probable or Clinically Definite ALS are widely used [17]. The Awaji Criteria expands on the EI Escorial criteria and include classification for Clinically Definite ALS, Clinically probable ALS, and Clinically possible ALS. Following the Awaji criteria for the diagnosis of ALS was associated with higher rates of being classified as definite or probable ALS. With the diagnosis of ALS being assigned many patients then have access to clinical trials [18].

\section{ALS Definition 1}

A diagnosis of ALS requires: 1) The presence of evidence of lower motor neuron degeneration by clinical, electrophysiological or neuropathologic examination; 2) Evidence of upper motor neuron degeneration by clinical examination and 3) Progressive spread of signs/symptoms from one region to another, as determined by history or examination; together with the absence of electrophysiological or pathological evidence of other disease processes that might explain the signs of lower motor neuron and/or upper motor neuron degeneration, and neuroimaging evidence of other disease processes that might explain the observed clinical and electrophysiological signs [19].

\section{ALS Definition 2}

Clinically definite amyotrophic lateral sclerosis is defined by clinical or electrophysiological evidence by the presence of lower motor neuron (LMN) as well as upper motor neuron (UMN) signs in the bulbar region and at least two spinal regions or the presence of $L M N$ and $U M N$ signs in three spinal regions. Clinically probable ALS is defined on clinical or electrophysiological evidence by LMN and UMN signs in at least two regions with some UMN signs necessarily rostral to (above) the LMN signs. The revised El Escorial Criteria have an additional category "Probable ALS-Laboratory Supported," which is defined when clinical signs of UMN and LMN dysfunction are found in only one region but electrophysiological signs of LMN loss are observed in two regions. Clinically possible ALS is defined when clinical or electrophysiological signs of UMN and LMN dysfunction are found in only one region or UMN signs are found alone in two regions or LMN signs are found rostral to UMN signs [18].

Electromyography changes were given the same diagnostic significance as clinical signs of lower motor neuron dysfunction in individual muscles. Fasciculations were taken as the equivalent to fibrillation potentials and positive sharp waves in recognition of denervation. Following the details of this diagnosis "An experienced clinician may be virtually certain of the diagnosis" in appropriate patient [20].

\section{ALS Definition 3}

Use of electrodiagnostic methods for 1) Establishment of diagnosis; 2) The differential diagnosis, and 3) Evaluation of quantitative progression. "Needle electromyography reveals active neurogenic changes over the wide territories of the body. Fibrillation potentials and positive sharp waves indicate presence of denervated fibers. However, they are not specific for ALS or even neurogenic process, and widespread occurrence of fibrillations are also characteristic for myositis and inclusion body myositis (IBM). Fasciculation potentials are spontaneous firing of the lower motor neuron and most of them are supposed to arise from the nerve endings. Fasciculation potentials are seen solely in neurogenic process and sufficiently specific for ALS" [21]. Fasciculation potentials are given the same significance as fibrillation potentials related to criteria for diagnosis of ALS.

The diagnosis of ALS remains a clinical one, based upon the presentation with progressive upper and lower motor neuron dysfunction, supported by electrophysiological studies. The diagnostic sensitivity has been a topic of debate [22,23]. Controversy about the disease exists in different hypothesis such as the "dying-forward" and the "dying-back" with no clear etiology being linked to the currently classified cases of ALS [24]. Considered one of the most devastating diseases known, there is little evidence for diagnosing and management of patients who are diagnosed with ALS [25].

Caregiver burden becomes a significant issue for a patient assigned a diagnosis of ALS. Worsening behavioral impairments along with the decreased physical ability are known to increase the caregiver burden [26]. Most literature indicating the differential diagnosis of ALS did not include the evaluation for cryoglobulinemia in the list of potential causes raising some intriguing points as related to this case. Other authors have found improved diagnosis with expanded testing of differential to discover alternate diagnosis resulting in beneficial therapy then being initiated [27].

\section{Definition and diagnosis of cryoglobulinemia vasculitis}

Cryoglobulins are immunoglobulins that precipitate as the serum is cooled below $37 \mathrm{C}^{\circ}$ and resolubilize when the serum is rewarmed [28]. Type I cryoglobulins are monoclonal immunoglobulins linked to a B-Cell lymphoproliferative 
Citation: Orehek AJ (2020) Obtaining Clinical Improvement in Amyotrophic Lateral Sclerosis with Stabilization of Progressive Functional Loss: Case Report Presentation and Review. J Neurodegener Disord 3(1):77-88

disorder. Type II cryoglobulins are monoclonal IgM immunoglobulins with rheumatoid factor activity and polyclonal IgG immunoglobulins. Type III cryoglobulins are polyclonal immunoglobulins of IgM and IgG. In the Type II cryoglobulins the IgM fraction has rheumatoid factor activity [29,30]. Type II cryoglobulins deposit as immune complex in the small vessels causing vasculitis resulting in purpura or leg ulcers and peripheral neuropathy or joint symptoms [31]. As found in this case the clinical presentation of purpura, muscle weakness, arthralgias, peripheral neuropathy, serum mixed cryoglobulins with rheumatoid factor activity, and low $\mathrm{C} 4$ has been well described as related to cryoglobulinemia vasculitis [32-34].

Diagnosis of cryoglobulinemia requires proper collection of the serum. Reports in the literature related to missed cases of cryoglobulinemia emphasize the challenges when dealing with this diagnosis $[35,36]$. Failure to properly separate serum from whole blood, lack of a water bath or warming plate during sample collection causes loss of cryoprecipitate due to temperature drop prior to centrifugation, inadequate volume of serum, collection of samples on patients who are on anticoagulants or improper incubation times are a few of the reasons the lab may report a false negative result [37]. Currently there are no internationally accepted standards for cryoglobulin detection methods and best practice approaches are developing. When evaluated it was found that only $36 \%$ of laboratories were using proper procedures for collection and processing of samples [38]. Proper collection requires that sample temperature does not drop below $37 \mathrm{C}$ until after separation of serum. Warm centrifugation should take place with temperature maintained at $37 \mathrm{C}$ to avoid false negative result. The serum should cryoprecipitate at $4 \mathrm{C}$ for three to seven days before being washed and resolubilized at $37 \mathrm{C}$. When challenges in obtaining a true positive lab result is combined with a failure to appreciate that even low levels of cryoglobulins can be associated with severe symptoms in some patients the diagnosis becomes demanding [39]. The detection of cryoglobulinemia in the patient with symptoms allows for the benefits associated with treatment of cryoglobulinemia vasculitis [40]. Diagnosis of cryoglobulinemia vasculitis is made when detectable cryoglobulinemia is associated with clinical manifestations of vasculitis [41].

\section{Definition and diagnosis of vasculitic neuropathy}

Patients may present with arthralgia, myalgia, peripheral neuropathy, renal involvement, cerebral vasculitis, purpura or cutaneous ulcers as examples. The peripheral neuropathy may present as mononeuritis multiplex, distal polyneuropathy or radiculopathy [42]. Vasculitic neuropathy are divided into nerve large arteriole vasculitis and nerve microvasculitis based upon the size of the blood vessels affected [43]. The nerve microvasculitis may overlap with the large arteriole vasculitis as involvement of the smallest arterioles and endoneurial capillaries cause damage of peripheral nerves. The peripheral nerve experiences ischemia to vasa nervorum resulting in the presentation of vasculitic neuropathy [44]. The identification and classification of vasculitic neuropathy by a variety of classification systems has added to the challenging and complex nature of this disorder [45]. Classically the histological evaluation of the sural nerve will demonstrate transmural inflammatory infiltrates of the vessel walls with fibrinoid necrosis and endothelial disruption. Diagnosis of vasculitic neuropathy with the finding of mononeuritis multiplex has been well established with the proper clinical history [46].

A hyper viscosity syndrome has been described as associated with the diagnosis of cryoglobulinemia. Similar findings in this case of cryoglobulins possessing the rheumoatid factor activity leading to hyperviscosity and clotting dysfunction have been published [47].

Additional factors affecting this case: Access to care and availability of medical coverage to the patient was a limiting factor. Once the family made the decision for medical treatment of cryoglobulinemia the patient faced additional challenges due to insurance coverage and insurance-healthcare system design. The "Diagnosis Related Groups" (DRG) is a complex financial system that involves both incentives and punishment to treating hospitals based upon efficiency of processing patients' discharges and avoiding any re-admission back to the hospital over a global period of time [48]. When a patient is discharged from a hospital but then re-admitted (same/similar diagnosis) within a month there are sanctions placed upon the hospital [49]. Clinically and practically this resulted in the patient having no access to a geographic hospital admission for related medical condition after she was discharged with the hyperviscosity syndrome and massive deep vein thrombosis through the 30 day global period. As this pattern of patient care is predictable additional medical care strategies needed to be employed [50].

During the phase of the case, predominated by hyperviscosity syndrome and severe cryoglobulinemia vasculitis causing severe vasculitic neuropathy, when the patient would have had the most benefit from plasma exchange or plasmapheresis she was unable to access this service [51-53]. Limiting patient's access to care also came from changes in the healthcare system where the patients prior access would have been to eight different geographically available hospitals, yet this has been reduced to four remaining [54]. One geographically available infusion lab (location where plasmapheresis is performed) was closed when the hospital was purchased by a larger corporation. Of the remaining geographic hospitals none of them completed plasmapheresis. Expanded geographic hospitals included at least four additional insurance company owned hospitals, with one of them offering the service but refused to provide to the patient due to patient age. Finally, an additional six hospitals were contacted at even further geographic distance (110-150 miles) and one of the six were able to offer plasmapheresis or plasma exchange. The available service hospital did not come to fruition because hospital indicated by telephone that the services would not be covered under the insurance plan of the patient due to unclear Medicare criteria and reimbursement issues (ICD-10-CM Code of D89.1 for diagnosis of cryoglobulinemia vasculitis listed as investigational in cases of amyotrophic lateral sclerosis) and the patient would have to cover the costs of the services at cash prices. (Healthcare Common Procedure Coding System 36514 for the procedure).

Limitation in access to specialists with long queue time be- 
Citation: Orehek AJ (2020) Obtaining Clinical Improvement in Amyotrophic Lateral Sclerosis with Stabilization of Progressive Functional Loss: Case Report Presentation and Review. J Neurodegener Disord 3(1):77-88

fore being provided with a future appointment impacted the case. The lack of prompt coordination while waiting for information to be processed for medical necessity, proper documentation, medical director record review of data, suitable ICD-10-CM codes to be evaluated by captious insurance corporations prior to approval, limited network specialist availability, and additional administrative factors adds to patient friction in attempts to streamline care. Compounding the effect is upon initiation of any treatment plan is the generally expected three month follow-up appointment, often resulting in too much time passing for the patient to make any accurate progress on their medical issues. In a setting of significant influx of "provider extenders" who may be qualified to provide services in that specialty area but may not have the same vast clinical experience of the given specialist results in challenges for patients with complex medical problems.

Inability for patient's story to be utilized during hospital admission where data in her electronic health record (EHR) contained important clinical reasoning details that were not integrated. Important details, as example hyperviscosity syndrome and massive deep vein thrombosis in the setting of positive cryoglobulins in the blood during a hospital admission were not evaluated or integrated by the hospital providers of medical care, and thus not evaluated or treated. Other authors have discussed how the use of EHR can obstruct clinicians' ability to treat complex cases because of fragmentation of patient data interconnections [55]. Often the hospital based providers of medical care have different priority starting with discharge planning at the time of the patient admission, but not priority of accurate diagnosis [56]. Large organizations owning geographic regions of access to care that do not have interoperable electronic health records resulted in duplication of testing at times, as example the patient had a number of CT scans of the brain during emergency room or hospital admission evaluation without any comparison to prior study [57].

Healthcare teams that referenced the patient's age as a factor in determination of care without evaluation of her individual medical patient story insert compounding factors into the care of the patient. Medical treatment of the patient over time has mitigated many of the leading causes of death in this patient [58]. As described above, the patient had complete revascularization of coronary arteries, adequate cardiac valve performance, no enlargement of ascending aorta or aortic root, and a completed evaluation of cardiac electrical system with treatment including dual chamber permanent pacemaker thus diminishing disease of the heart. Evaluation of malignant neoplasms have included esophagogastroduodenoscopy, colonoscopy, mammography, skin evaluation and successful treatment of breast cancer. Medical error as the third leading cause of death in the United States remains unmitigated. Complex factors exist of how medical care is provided in the United States, but it would be bewildering that health care teams denying care to the patient would be most concerned of medical errors by their hand directly leading to adverse outcome as the primary reason for care determination [59]. Stroke, often listed as fifth most common cause of mortality, was mitigated across a wide range of potential etiol- ogy [60]. As described in this case: complete evaluation of prothrombotic state by treatment of genetic defects as reviewed in Figure 9, carotid arteries had identified stenosis in the $1-19 \%$ range, atrial fibrillation would be identified during any interrogation of the pacemaker and she remains on anticoagulation, valvular heart disease is minimal, there is no patent foramen ovale, aortic arch did not have significant atherosclerotic plaques. As evaluated these details and evaluation would lead to reduction in ischemic stroke by multiple mechanisms. The patient remains at risk of ischemic stroke from vasculitis and hyperviscosity syndrome, yet healthcare teams denying patient treatment due to a risk that is the primary etiology of treatment plan is perplexing. Alzheimer's disease is unlikely in this patient who has scores as shown in Figure 13, despite the CSF data of A-beta 42 to T-tau index (ATI) 0.45 with interpretation: Alzheimer Disease. The patient did not have diabetes mellitus. Kidney disease is always a future potential, however, during the evaluation of her medical needs her creatinine 0.75 $\mathrm{mg} / \mathrm{dl}$ remained in a normal range. The patient did not have any evidence of liver disease or cirrhosis. Blood pressure has been under good control over the last decade with systolic blood pressure being under $119 \mathrm{~mm} / \mathrm{Hg}$. The patient did not have any evidence of Parkinson's disease. Remaining mortality would be unresolved infections, yet with proper medical care this should not be a determining factor as age is related to medical care. Other authors have written about health care disparities that could be applicable to this situation [61].

The effects of treatments the patient received during the course of the illness could have mitigated or altered the disease in course. As example, the use of hydroxychloroquine, steroids, methotrexate (both oral and IM) and meloxicam could have altered aspects of progression or presentation.

\section{Conclusion}

Designated as amyotrophic lateral sclerosis and considered a "diagnosis of exclusion" the complex physiological interactions resulting in neuron damage raises a concern when not all etiology have been excluded. The use of ever changing classification systems to describe the neurological manifestations of damage to the nervous system will not bring the same accuracy in treatment that identification of the etiology(s) will provide [62]. As examples of diseases designated by way of "diagnosis of exclusion": acephalgic migraine, dementia lacking distinct histopathologic features, frontotemporal dementia, vascular dementia, dementia with Lewy bodies, movement disorder, optic neuritis, normal pressure hydrocephalus, multiple sclerosis, frontotemporal dementia, or Alzheimer's disease all lack etiology as cause of diagnosis. When a complete and accurate evaluation identifies the etiology of damage to the neurons then the diagnosis is based upon the reason for the damage. Improving the diagnosis based upon etiology allows strategy and treatment to be directed. Consider neuron loss based upon vasculitis, sarcoidosis, hypoxia, micron stroke $[63,64]$, systemic lupus erythematosis, hypercoaguable state or Lyme disease and how treatment strategy can become most effective [63]. There is innate danger when clinical manifestations are used in assigning a diagnosis based upon an ever changing classification system. 
Citation: Orehek AJ (2020) Obtaining Clinical Improvement in Amyotrophic Lateral Sclerosis with Stabilization of Progressive Functional Loss: Case Report Presentation and Review. J Neurodegener Disord 3(1):77-88

\section{Declarations}

Ethics approval and consent to participate Signed patient consent on file. Consent for publication Signed patient consent on file. Availability of data and materials Data and material available for review.

\section{Competing Interest}

There are no competing interests.

\section{Funding}

There was no funding.

\section{Authors Contributions}

A J. Orehek was responsible for the manuscript and all research including final preparations.

\section{Acknowledgement}

None.

\section{References}

1. Midroni Gyl, Juan M Bilbao (1995) Peripheral neuropathy and the role of nerve biopsy. Biopsy diagnosis of peripheral neuropathyp 1-11.

2. England JD, Gronseth GS, Franklin G, et al. (2009) Evaluation of distal symmetric polyneuropathy: The role of autonomic testing, nerve biopsy, and skin biopsy (an evidence-based review). Muscle Nerve 39: 106-115.

3. Davies L, Spies JM, Pollard JD, et al. (1996) Vasculitis confined to peripheral nerves. Brain 119: 1441-1448.

4. Lacomis D (2002) Small-fiber neuropathy. Muscle Nerve 26: 173188.

5. Montero-Odasso M, Almeida QJ, Bherer L, et al. (2018) Consensus on shared measures of mobility and cognition: From the Canadian consortium on neurodegeneration in aging (CCNA). J Gerontol A Biol Sci Med Sci.

6. Nasreddine ZS, Phillips NA, Bédirian V, et al. (2005) The montreal cognitive assessment, MoCA: A brief screening tool for mild cognitive impairment. J Am Geriatr Soc 53: 695-699.

7. Cumming TB, Churilov L, Lindén T, et al. (2013) Montreal cognitive assessment and mini-mental state examination are both valid cognitive tools in stroke. Acta Neurol Scand 128: 122-129.

8. Noone P (2015) Addenbrooke's cognitive examination-III. Occupational Medicine 65: 418-420.

9. Powsner S, Powsner D (2005) Cognition, copyright, and the classroom. Am J Psychiatry 162: 627-628.

10. Hodkinson HM (1972) Evaluation of a mental test score for assessment of mental impairment in the elderly. Age Ageing 1: 233-238.

11. Brodaty H, Pond D, Kemp NM, et al. (2002) The GPCOG: A new screening test for dementia designed for general practice. J Am Geriatr Soc 50: 530-534.

12. Hidler J, Brennan D, Black I, et al. (2011) ZeroG: Overground gait and balance training system. J Rehabil Res Dev 48: 287-98.

13. R.G. Miller, JA Rosenberg, DF Gelinas, et al. (1999) Practice parameter: The care of the patient with amyotrophic lateral sclerosis (an evidence-based review): Report of the quality standards subcommittee of the american academy of neurology :The ALS Practice Parameters Task Force. Neurology 52: 1311-1323.

14. Kiernan MC1, Vucic S, Cheah BC, et al. (2011) Amyotrophic lateral sclerosis. Lancet 377: 942-55.

15. Brown RH, Al-Chalabi A (2017) Amyotrophic lateral sclerosis. N Engl J Med 377: 162-172.

16. Mitsumoto H (2017) Guidelines for ALS clinical trials (AIRLIE HOUSE 2016) and clinical criteria (The diagnosis WFN research group 2015- A critical appraisal). Journal of the Neurological Sciences 381.

17. Agosta F, Al-Chalabi A, Filippi M, et al. (2014) The El Escorial criteria: Strengths and weaknesses. Amyotroph Lateral Scler Frontotemporal Degener 16: 1-7.

18. Costa J, Swash M, Carvalho MD (2012) Awaji criteria for the diagnosis of amyotrophic lateral sclerosis. Arch Neurol 69: 1410-6.

19. Brooks BR, Miller RG, Swash M, et al. (2000) El Escorial revisited: Revised criteria for the diagnosis of amyotrophic lateral sclerosis. Amyotroph Lateral Scler Other Motor Neuron Disord 1: 293-299.

20. Carvalho M , Dengler R, Eisen A, et al .(2008) Electrodiagnostic criteria for diagnosis of ALS . Clin Neurophysiol 119: 497-503

21. Sonoo M, Higashihara M, Hokkoku K (2011) Electrodiagnosis of ALS: Its practical aspects. Rinsho Shinkeigaku 51: 1111-1113.

22. Carvalho MD, Swash M (2009) Awaji diagnostic algorithm increases sensitivity of El Escorial criteria for ALS diagnosis. Amyotroph Lateral Scler 10: 53-57.

23. Li D, Liu M, Cui B, et al. (2017) The Awaji criteria increases the diagnostic sensitivity of the revised El Escorial criteria for amyotrophic lateral sclerosis diagnosis in a Chinese population. PLoS One 12: e0171522.

24. Kiernan MC, Steve Vucic, Benjamin C Cheah et al. (2011) Amyotrophic lateral sclerosis. The Lancet 377: 942-955.

25. Inghilleri Maurizio, Elisa lacovelli (2011) Clinical neurophysiology in ALS. Arch Ital Biol 149: 57-63.

26. De Wit Jessica, Leonhard A Bakker, Annerieke $C$ van Groenestijn et al. (2018) Caregiver burden in amyotrophic lateral sclerosis: A systematic review.Palliat med 32: 231-245.

27. Davenport RJ, Swingler RJ, Chancellor AM, et al. (1996) Avoiding false positive diagnoses of motor neuron disease: lessons from the Scottish Motor Neuron Disease Register. J Neurol Neurosurg Psychiatry 60: 147-151.

28. Brouet JC, Clauvel JP, Danon F, et al. (1974) Biologic and clinical significance of cryoglobulins: A report of 86 cases. Am J Med 57: 775-788.

29. Motyckova G, Murali M (2011) Laboratory testing for cryoglobulins. Am J Hematol 86: 500-502.

30. Cacoub P, Comarmond C, Domont F, et al. (2015) Cryoglobulinemia vasculitis. The American journal of medicine 128: 950-955.

31. Damoiseaux J (2014) The diagnosis and classification of the cryoglobulinemic syndrome. Autoimmunity reviews 13: 359-362.

32. Ferri C, Antonelli A, Mascia MT, et al. (2007) B-cells and mixed cryoglobulinemia. Autoimmun Rev 7: 114-120.

33. Garcin R, Mallarmé J, Rondot $P$ (1957) Cryoglobulinémie et névrite multiple des membres inférieurs. Rev Neurol (Paris) 97: 142-146.

34. LoSpalluto J, Dorward B, Miller W (1962) Cryoglobulinemia 
Citation: Orehek AJ (2020) Obtaining Clinical Improvement in Amyotrophic Lateral Sclerosis with Stabilization of Progressive Functional Loss: Case Report Presentation and Review. J Neurodegener Disord 3(1):77-88

based on interaction between a gamma macroglobulin and $7 \mathrm{~S}$ gamma globulin. The American journal of medicine 32: 142-147.

35. Gulli F, Santini SA, Napodano C, et al. (2017) Cryoglobulin test and cryoglobulinemia Hepatitis C-Virus related. Mediterr J Hematol Infect Dis 9: e2017007.

36. Kay J, McCluskey RT (2005) Case 31-2005-A 60-year-old man with skin lesions and renal insufficiency. New England Journal of Medicine 353: 1605-1613.

37. Kallemuchikkal U, Gorevic P D (1999) Evaluation of cryoglobulins. Archives of pathology and laboratory medicine 123: 119125.

38. Vermeersch P, Gijbels K, Mariën G, et al. (2008) A critical appraisal of current practice in the detection, analysis, and reporting of cryoglobulins. Clin Chem 54: 39-43.

39. Sargur R, White $P$, Egner W (2010) Cryoglobulin evaluation: best practice? Ann Clin Biochem 47: 8-16.

40. Cacoub P, Comarmond C, Domont F, et al. (2015) Cryoglobulinemia vasculitis Am J Med 128: 950-955.

41. Terrier B, Krastinova E, Marie I, et al. (2012) Management of noninfectious mixed cryoglobulinemia vasculitis: data from 242 cases included in the CryoVas survey. Blood 119: 5996-6004.

42. Schaublin GA, Michet Jr CJ, Dyck PJB, et al. (2005) An update on the classification and treatment of vasculitic neurol. Lancet Neurology 4: 853-865.

43. Gwathmey KG, Burns TM, Collins MP, et al. (2014) Vasculitic neuropathies. Lancet Neurol 13: 67-82.

44. Nemni R, Corbo M, Fazio R, et al. (1988) Cryoglobulinaemic neuropathy: A Clinical, Morphological And Immunocy Tochemical Study Of 8 Cases. Brain 111: 541-552.

45. Burns TM, Schaublin GA, Dyck PJ B (2007) Vasculitic neuropathies. Neurologic clinics 25: 89-113.

46. Agadi JB, Raghav G, Mahadevan A, et al. (2012) Usefulness of superficial peroneal nerve/peroneus brevis muscle biopsy in the diagnosis of vasculitic neuropathy. Journal of Clinical Neuroscience 19: 1392-1396.

47. Meltzer M, Franklin EC, Elias K, et al. (1966) Cryoglobulinemia-a clinical and laboratory study: II. Cryoglobulins with rheumatoid factor activity. The American journal of medicine 40: 837-856.

48. https://www.cms.gov/Medicare/Medicare-Fee-for-Service-Payment/AcutelnpatientPPS/MS-DRG-Classifications-and-Software. html

49. Federal Register Volume 83, Number 160 (Friday, August 17,
2018), Rules and Regulations, Pages $41144-41784$, From the Federal Register Online via the Government Publishing Office.

50. Fonarow GC (2018) Unintended harm associated with the hospital readmissions reduction program. JAMA 320: 2539-2541.

51. Berkman EM, Orlin JB (1980) Use of plasmapheresis and partial plasma exchange in the management of patients with cryoglobulinemia. Transfusion 20: 171-178.

52. Bombardieri S, Maggiore Q, L'abbate, et al. (1981) Plasma exchange in essential mixed cryoglobulinemia. Plasma Therapy 2(10).

53. McGovern TW, Enzenauer RJ, Fitzpatrick JE (1996) Treatment of recalcitrant leg ulcers in cryoglobulinemia types I and II with plasmapheresis. Arch dermatol 132: 498-500.

54. RURAL HOSPITAL CLOSURES (2018) Number and Characteristics of Affected Hospitals and Contributing Factors.GAO-18-634: Published: Aug 29, 2018. Publicly Released: Sept 28, 2018. (accessed 3/25/2019).

55. Varpio L, Rashotte J, Day K, et al. (2015) The EHR and building the patient's story: A qualitative investigation of how EHR use obstructs a vital clinical activity. Int J Med Inform 84: 1019-1028.

56. Stevens JP, Nyweide DJ, Maresh S, et al. (2017) Comparison of hospital resource use and outcomes among hospitalists, primary care physicians, and other generalists. JAMA Intern Med 177: 1781-1787.

57. Madden JM, Lakoma MD, Rusinak D, et al. (2016) Missing clinical and behavioral health data in a large electronic health record (EHR) system. J Am Med Inform Assoc 23: 1143-1149.

58. Xu J, Murphy SL, Kochanek KD, et al. (2018) Deaths: Final data for 2016. Natl Vital Stat Rep 67: 1-75

59. Makary MA, Daniel M (2016) Medical error-the third leading cause of death in the US. BMJ 353: i2139.

60. Panel Mohr JP, Albers GW, Amarenco P, et al. (1997) Etiology of stroke. Stroke 28: 1501-1506.

61. Roberts ET, Zaslavsky AM,McWilliams JM (2018) The value-based payment modifier: program outcomes and implications for disparities. Ann Intern Med 168: 255-265.

62. Thompson AJ, Banwell BL, Barkhof F, et al. (2018) Diagnosis of multiple sclerosis: 2017 revisions of the McDonald criteria. Lancet Neurol, 17: 162-173.

63. Rabiee N, Ahmadi S, Afshari R, et al. (2020) Polymeric Nanoparticles for Nasal Drug Delivery to the Brain: Relevance to Alzheimer's Disease. Adv Ther 2000076.

64. Hamblin MR (2019) Photobiomodulation for Alzheimer's Disease: Has the Light Dawned? Photonics 6:77.

DOI: $10.36959 / 459 / 602$

Copyright: (C) 2020 Orehek AJ. This is an open-access article distributed under the terms of the Creative Commons Attribution License, which permits unrestricted use, distribution, and reproduction in any medium, provided the original author and source are credited. 